novative way to apply wilderness medicine skill sets to global health education in medical school in order to best prepare students to work effectively in resource-poor settings.

Amita A. Kulkarni

Liz B. Francis

Jay M. Lemery, MD

Dana L. Sacco, MD

Manhattan, NY, USA

\section{A Prospective Cohort Study of Acute Kidney Injury in Multi-Day Ultramarathon Runners}

Objective.-Ultramarathon foot races of distances greater than the traditional 26.2 mile marathon are increasing in popularity, attracting more than 70000 annual participants worldwide. Prior studies have consistently documented renal function impairment, but only after race completion. The incidence of renal injury during these multi-day ultramarathons is currently unknown. This is the first prospective cohort study to evaluate the incidence of acute kidney injury (AKI) in runners during a multi-day ultramarathon foot race.

Methods.-Demographic and biochemical data gathered via phlebotomy and analyzed by iSTAT (Abbott, NJ) were collected at the start and finish of Day 1 (25 miles), Day 3 (75 miles), and Day 5 (140 miles) during Racing The Planet's 150-mile, 7-day self-supported desert ultramarathons. Pre-established RIFLE criteria, using creatinine $(\mathrm{Cr})$ and glomerular filtration rate (GFR), defined AKI as "No Injury" $(\mathrm{Cr}<1.5 \times$ normal, decrease of GFR $<25 \%)$, "Risk" $(\mathrm{Cr} 1.5 \times$ normal, decrease of GFR by $25 \%-49 \%)$, and "Injury" (Cr $2 \times$ normal, decrease of GFR by $50 \%-75 \%$ ).

Results. - Thirty racers, $76 \%$ male with a mean ( \pm SD) age of $39 \pm 10$ years, were studied during the 2008 Sahara $(n=7$, 23.3\%), 2008 Gobi $(n=10,33 \%)$, and 2009 Namibia $(n=13$, $43.3 \%)$ events. The average decrease in GFR from Day 1 start to Day 1 finish was $28 \pm 25(P<.0001,95 \%$ CI, 18.5-37.6); Day 1 start to Day 3 finish was $29.6 \pm 20.1(P<.0001,95 \%$ CI, 18.4-40.7); and Day 1 start to Day 5 finish was $30.9 \pm 17.5$ $(P<.0001,95 \%$ CI, 20.8-41). Runners categorized as Risk and Injury for AKI after Stage 1 was $44.8 \%$ and $10 \%$; after Stage $3,67 \%$ and $13 \%$; and after Stage $5,57.1 \%$ and $7.1 \%$.

Conclusions.-The majority of participants developed significant levels of renal impairment. Given the changes in renal function, potentially harmful nonsteroidal anti-inflammatory drugs should be minimized to prevent exacerbating acute kidney injury.

Grant S. Lipman, MD Garrett Chan, PhD Sarah Logan, MD Palo Alto, CA, USA

Brian Krabak, MD Seattle, WA, USA

Brandee Waite, MD Anil Menon, MD

Sacramento, CA, USA

\section{Who is Being Rescued in Canadian National Parks?}

Introduction.-There has been a growing body of literature reviewing the epidemiology of injuries, illnesses, and search and rescue (SAR) operations in National Parks in the United States. There are 42 National Parks in Canada that experienced over 12 million visitors in 2010. Little research has been published to date on SAR trends in Canadian National Parks.

Objective.-To review the current literature regarding SAR operations in Canadian National Parks and assess the need for further research in this area.

Methods.-Articles in the MEDLINE database were identified that analyzed SAR operations or injury and illnesses occurring in Canadian National Parks. The Parks Canada website was reviewed for relevant information available to the public regarding safety and SAR operations in National Parks.

Results.-One peer-reviewed article was identified that analyzed the epidemiology of SAR operations in Banff, Yoho, and Kootenay National Parks. No further relevant articles were found. From 1994 to 1998, Parks Canada reported a yearly average of 1400 safety incidents in National Parks. Pacific Rim National Park Reserve (PRNPR) is reported to experience the most SAR operations from all National Parks, with the West Coast Trail accounting for 80 to 100 incidents annually. There were 20 accident reports available from the 7 Mountain National Parks in 2010, with 4 self-reported "near-miss" accidents.

Conclusions.-A paucity of data exists regarding SAR operations in Canadian National Parks. Epidemiological studies conducted in the United States may not be extrapolated to Canada due to possible differences in climate, activity rates, and safety programs. A recent study of US National Parks demonstrated the most common SAR environment was the mountain environment, whereas in Canada it is reported that PRNPR, a coastal environment, experiences the highest number of SAR operations yearly. Further research into these incidents is needed before critical analysis and improvement of prevention strategies targeting high-risk areas and activities can be made.

Jonathan A. Malo, MBBS

Townsville, Queensland, Australia

Eiman Zargaran, MD

North Vancouver, British Columbia, Canada

\section{Injury and Illness on the West Coast Trail: Still the graveyard of the Pacific?}

Introduction.-The West Coast Trail (WCT) is a $75 \mathrm{~km}$ trek in Pacific Rim National Park Reserve on the southwest coast of Vancouver Island, British Columbia, with 4000 to 6000 hikers attempting to complete the trail annually. It is a physically demanding and strenuous hike renowned for its unique infrastructures and violent weather. Parks Canada quotes that $1 \%$ to $2 \%$ of hikers require evacuation every year, while an additional 200 sustain minor injuries. There is growing literature reviewing injuries and illnesses in US National Parks that consistently show hikers to be the most common group requiring medical 
assistance. To date, no formal studies have analyzed the morbidity associated with a specific hike where accurate census data is available.

Objective.-To review the epidemiology of injuries, illnesses, and causes of evacuations on the WCT and make recommendations for improving participant safety.

Methods.-Parks Canada staff provided de-identified public safety occurrence data within the WCT from 2005 to 2010. These reports were retrospectively reviewed and the relevant data was extracted. Only reports involving hikers during the May 1st to September 30th hiking season were included. The 2-proportion z-test was used to determine statistical significance.

Results.-We reviewed 457 occurrence reports, of which 310 met inclusion criteria, corresponding to an incident rate of 1 per 104 hikers. This was significantly increased $(P<.01)$ from the rate of 1 in 220 hikers that Parks Canada stated required assistance in 1993 to 1994. Evacuation was reported in $196(63 \%)$ cases, while only $56(18 \%)$ documented administration of first aid. Injuries and/or illnesses were reported in 89 and 22 of the cases, respectively. Sixty-seven (21.6\%) incident reports originated from $\mathrm{km} 70$ to 75 of the trail.

Conclusions.-There was an alarmingly high number of evacuations and morbidity on the WCT given the limited number of hikers. Injuries and illnesses were likely underreported. Further analysis is required to determine causes for the significantly increased incident rate found in our study compared to incident rates in 1993 to 1994. More detailed injury surveillance is required for further assessment of morbidity on the WCT. This will allow for appropriate prevention strategies to be implemented.

Jonathan A. Malo, MBBS Townsville, Queensland, Australia

Eiman Zargaran, MD North Vancouver, British Columbia, Canada

\section{Impact of Handheld SONAR Devices on SCUBA Diver Rescue}

Objective.-To investigate if a handheld sound navigation ranging (SONAR) device significantly reduces the median duration to locate a missing diver. A secondary objective is to determine the effect a rescue adjunct has on the participant's confidence in performing a search for a missing diver.

Methods.-This Internal Review Board-approved, prospective, crossover study used a voluntary convenience sample of 10 self-contained underwater breathing apparatus (SCUBA) divers. Participants conducted both a standard and modified search to locate a simulated, missing diver. The standard search utilized a conventional search pattern starting at the point where the missing diver (simulated) was last seen. The modified search used a SONAR beacon to augment the search. For each search method, successful completion of the search was defined as locating the missing diver within 40 minutes.

Results.-Twenty total dives were completed. Using a standard search pattern, only 1 diver (10\%) found the missing diver, taking 18 minutes and 45 second. In the SONAR-assisted search group, all 10 participants (100\%) found the missing diver, taking an average of 2 minutes and 47 seconds (SD 1 minute 20 seconds). Using the Nonparametric Related Samples Wilcoxon Signed Rank Test, actual times between the SONAR group and the standard group were significant $(P<.01)$. Using paired samples $t$-tests, the SONAR group's self-assessed confidence increased significantly after using the SONAR $(P<$ $.001)$, whereas the standard group decreased in confidence $(P=0.111)$.

Conclusions.-Handheld SONAR significantly reduces the median duration to locate a missing diver as well as increasing user confidence in their ability to find a missing diver when compared to standard search and rescue techniques.

Owen McGrane, MD

Aaron Cronin, PA-C

David Hile, MD

Tacoma, WA, USA

\section{Implications of Parental Influence on Child/Adolescent Helmet Use in Snow Sports}

Objective.-Helmets have become increasingly more important with the advent of extreme snow sports, including skiing and snowboarding. Despite proven benefits, the rate of helmet use is less than ideal. The objective of this study was to describe the factors that influence helmet use among children who participate in skiing and/or snowboarding.

Methods.- Voluntary surveys were distributed to children/ adolescents between the ages of 6 and 17 years old (and their parents) at a major Colorado ski resort during the 2006-2007 and 2007-2008 seasons. The survey included questions related to demographics, child/adolescent helmet use, parent helmet use, and reasons for wearing or not wearing a helmet. Logistic regression was used to determine whether parent helmet use, gender, and/or age were related to child helmet use.

Results.-A total of 206 children/adolescents completed the survey. Fifty one percent of the respondents were male and $49 \%$ were female. The proportion of children/adolescents and parents that reported they always wear a helmet was $83 \%$ and $53 \%$, respectively. Among children/adolescents, safety was the most frequent reason $(87.7 \%)$ given for wearing a helmet. The most common reason for not wearing a ski/snowboard helmet was comfort $(48.6 \%)$. There was a significant relationship between parental helmet use and child helmet use $(P<.0001)$. The odds of a child wearing his/her helmet are 9.5 times higher (95\% CI, 3.5-2.8) if their parents wear a helmet than if their parents do not wear a helmet. Gender and age were not significantly $(P>.05)$ related to child helmet use.

Conclusions.-The results demonstrate the overwhelming influence that parental helmet use has on their child/adolescent's decision to wear a helmet. Physician-based interventions that are targeted at increasing helmet use should focus on educating the parents about both the protective value of the helmet and, equally important, the role-modeling effect helmet use will have on their children. 